\title{
A superheated Bose-condensed gas
}

\author{
Alexander L. Gaunt ${ }^{\dagger}$, Richard J. Fletcher ${ }^{\dagger}$, Robert P. Smith` and Zoran Hadzibabic
}

Our understanding of various states of matter usually relies on the assumption of thermodynamic equilibrium. However, the transitions between different phases of matter can be strongly affected by non-equilibrium phenomena. Here we demonstrate and explain an example of non-equilibrium stalling of a continuous, second-order phase transition. We create a superheated atomic Bose gas, in which a Bose-Einstein condensate (BEC) persists above the equilibrium critical temperature ${ }^{1,2}, T_{c}$ if its coupling to the surrounding thermal bath is reduced by tuning interatomic interactions. For vanishing interactions the BEC persists in the superheated regime for a minute. However, if strong interactions are suddenly turned on, it rapidly boils away. Our observations can be understood within a twofluid picture, treating the condensed and thermal components of the gas as separate equilibrium systems with a tunable inter-component coupling. We experimentally reconstruct a non-equilibrium phase diagram of our gas, and theoretically reproduce its main features.

Non-equilibrium many-body states can persist for a very long time if, for example, a system is integrable, the transition to the lower free-energy state is inhibited by an energy barrier, or the target equilibrium state is continuously evolving owing to dissipation. Ultracold atomic gases offer excellent possibilities for fundamental studies of non-equilibrium phenomena ${ }^{3-14}$ and have been used to create counter-intuitive states such as repulsively bound atom pairs ${ }^{5}$ and Mott insulators with attractive inter-particle interactions ${ }^{12}$.

Our superheated Bose gas is reminiscent of superheated distilled water, which remains liquid above $100^{\circ} \mathrm{C}$. Specifically, as the temperature characterizing the average energy per particle and the populations of the excited states rises above $T_{c}$, the cloud remains in the partially condensed phase, which in true equilibrium should exist only below $T_{\mathrm{c}}$. However, there are also important differences. Boiling of water is a first-order phase transition and is stalled in clean samples by the absence of nucleation centres. In that case the transition is inhibited by an energy barrier. For a second-order phase transition such a barrier does not exist and the superheating we observe is a purely dynamical non-equilibrium effect, which arises because different properties of the system evolve at different rates. In this respect our gas also bears resemblance to the long-lived non-equilibrium spin structures observed in spinor condensates ${ }^{6,9}$, pre-thermalized states in quenched onedimensional Bose gases ${ }^{14}$ and supercritical superfluids predicted to occur in quenched two-dimensional gases ${ }^{15}$. In all of those cases, however, non-equilibrium states are observed owing to the system's slow approach to true equilibrium. Here, the system actually evolves away from equilibrium.

In Fig. 1 we summarize the basic idea of our experiments and the key concepts needed to understand them. In an equilibrium gas, a BEC is present only if $T<T_{\mathrm{c}}$, where $T_{\mathrm{c}}$ depends on the total particle number $N$, or equivalently if the chemical potential $\mu>\mu_{\mathrm{c}}$. In a standard experiment, after a BEC is produced, it gradually decays because $T$ rises, owing to technical heating, and/or $T_{\mathrm{c}}$ decreases, because $N$ decays through various inelastic processes. As $T / T_{\mathrm{c}}$ increases, elastic collisions redistribute the atoms between the thermal and condensed components, aiming to ensure the equilibrium particle distribution. The BEC atom number, $N_{0}$, can therefore decay in two ways: by direct inelastic loss, and through elastic transfer of atoms into the thermal component. Here we reduce the rate of the elastic particle transfer by tuning the strength of inter-particle interactions, characterized by the s-wave scattering length $a$. This protects the BEC deep into the superheated regime, where $N_{0}>0$ even though $T>T_{\mathrm{c}}$.

We can understand our observations within the two-fluid picture outlined in Fig. 1b. Here we treat the thermal and condensed components as two coupled subsystems with atom numbers $N^{\prime}$ and $N_{0}$, chemical potentials $\mu^{\prime}$ and $\mu_{0}$, and instantaneous per-particle inelastic decay rates $\Gamma^{\prime}$ and $\Gamma_{0}$, respectively. In equilibrium $\mu^{\prime}=\mu_{0}$; note that $\mu_{0}$ is defined only if $N_{0}>0$, so $\mu_{0}>\mu_{\mathrm{c}}$.

The two components are coupled in two ways, both dependent on the scattering length $a$. First, the local kinetic thermal equilibrium between the collective excitations in the BEC (phonons) and the thermal bath is ensured by Landau damping, the rate of which is $\propto \sqrt{a}$ (refs 16,17). Second, the global phase equilibrium (that is, the equilibrium condensed fraction $N_{0} / N$ ) is ensured by the elastic scattering with a rate $\propto a^{2}$. Crucially, owing to the different scalings with $a$, we find a large parameter space where the two components can be considered to be in kinetic equilibrium while the system is not in global phase equilibrium. In other words, the two components are at the same temperature, but have different chemical potentials.

In our optically trapped ${ }^{39} \mathrm{~K}$ gas ${ }^{18}$, we control $a$ by an external magnetic field tuned close to a Feshbach resonance at $402 \mathrm{G}$ (ref. 19), the dominant source of $\Gamma^{\prime}$ and $\Gamma_{0}$ is spontaneous scattering of photons from the trapping laser beams, and $\Gamma_{0}$ has an additional contribution from three-body recombination.

The key steps in our experimental sequence are summarized in Fig. 1c. We start by preparing a partially condensed gas in the $\left|F, m_{F}\right\rangle=|1,1\rangle$ hyperfine ground state by evaporative cooling at $a=135 a_{0}$, where $a_{0}$ is the Bohr radius ${ }^{18}$. We then reduce $a$ (over $50 \mathrm{~ms}$ ) and follow the subsequent evolution of the cloud, probing the atomic momentum distribution by absorption imaging in time-of-flight expansion. Reducing $a$ (at constant $N_{0}$ ) initially reduces $\mu_{0}$ below $\mu^{\prime}$ (ref. 13), but subsequently $\mu_{0}$ decays slower.

In Fig. 2 we quantitatively contrast the equilibrium evolution of a cloud at $a=83 a_{0}$ and the non-equilibrium evolution at $5 a_{0}$. In both cases we start at time $t=0$ (Fig. 1c) with $N_{0} \approx 2 \times 10^{4}$ and $N \approx 2 \times 10^{5}$ at $T \approx 160 \mathrm{nK}$. In both cases $T_{\mathrm{c}}$ decreases at a similar rate owing to similar $N$ decay. At $5 a_{0}$, the temperature rises faster owing to less effective evaporative cooling at a fixed optical trap depth.

Whether the gas is in equilibrium or not, it can always be characterized by two extensive variables, the total particle number $N$ and energy $E$. We measure these quantities by direct summation 

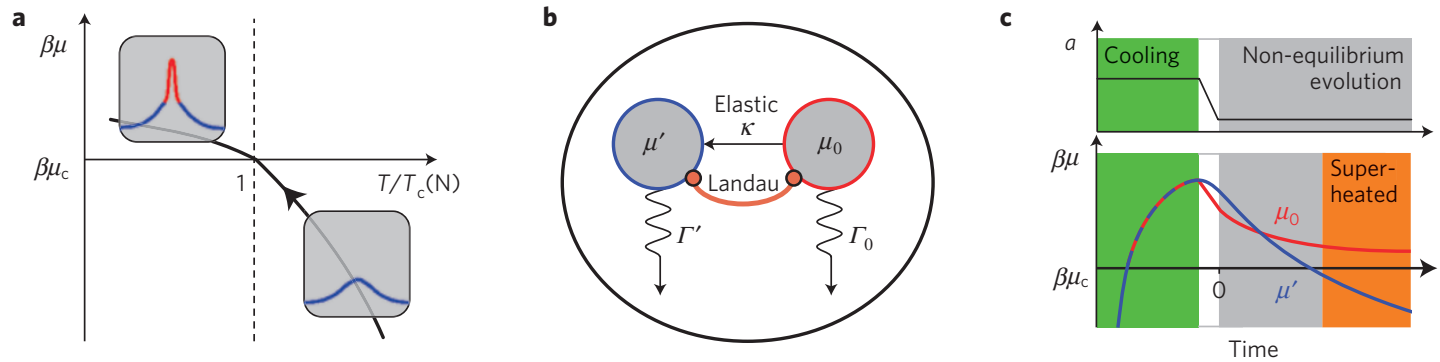

Figure 1 | Creating and understanding a superheated Bose-condensed gas. a, In equilibrium, a BEC is present if $T<T_{\mathrm{C}}$ or equivalently $\mu>\mu_{\mathrm{C}}$ (here, $\beta=1 /\left(k_{\mathrm{B}} T\right)$ ). The arrow indicates the cooling trajectory along which a $\mathrm{BEC}$ is produced. The insets show measured momentum distributions, with the condensed component indicated in red. $\mathbf{b}$, Two-component picture. The thermal and condensed components have chemical potentials $\mu^{\prime}$ and $\mu_{0}$, and inelastic decay rates $\Gamma^{\prime}$ and $\Gamma_{0}$, respectively. The net flow of particles between the two components, $\kappa$, depends on $\mu^{\prime}$, $\mu_{0}$ and the scattering rate $\propto a^{2}$. In equilibrium $\mu^{\prime}=\mu_{0}=\mu^{\mathrm{eq}}$. The Landau damping of the collective modes in the BEC has a rate $\propto \sqrt{a}$. c, Time sequence of the experiment. Reducing $a$ after preparing a BEC reduces the coupling between the two components and extends the condensate lifetime. In the superheated regime $\mu^{\prime}<\mu^{\text {eq }}<\mu_{\mathrm{c}}$ but $\mu_{0}>\mu_{c}$.
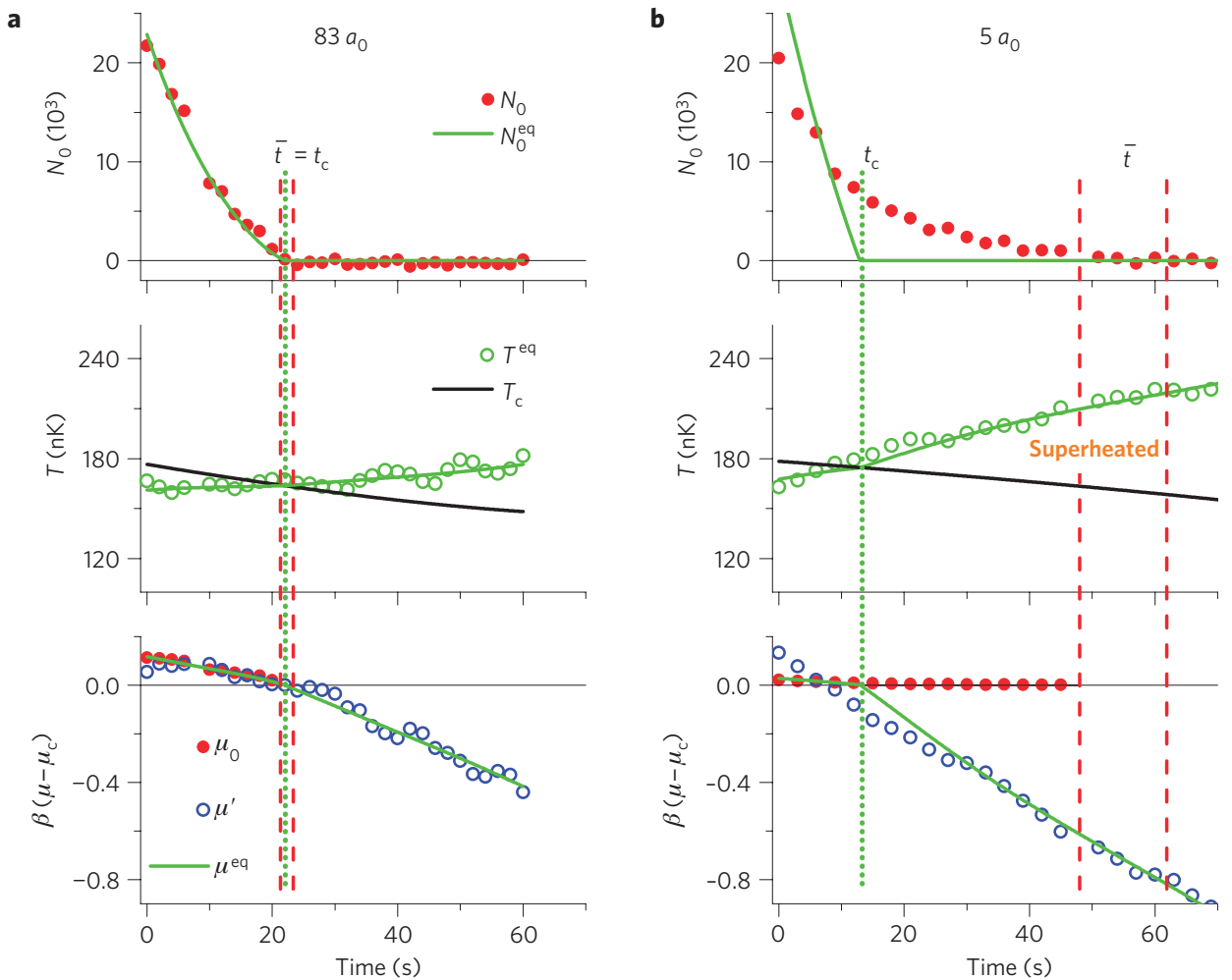

Figure 2 | Equilibrium versus non-equilibrium BEC decay. a, At $a=83 a_{0}$ the cloud is always in quasi-static equilibrium. The measured $N_{0}$ is in excellent agreement with the predicted $N_{0}^{\mathrm{eq}}$ and vanishes when $T^{\mathrm{eq}}=T_{c}$; the three separately calculated chemical potentials, $\mu_{0}, \mu^{\prime}$ and $\mu^{\mathrm{eq}}$, all agree with each other. The dotted green line marks the equilibrium critical time, $t_{c}$, and the dashed red lines show the experimental bounds on the time $\bar{t}$ when the BEC actually vanishes. $\mathbf{b}$, At $5 a_{0}$, the BEC persists in the superheated regime $\left(T^{\mathrm{eq}}>T_{\mathrm{c}}\right)$ for $\bar{t}-t_{\mathrm{c}} \approx 40 \mathrm{~s}$

of the momentum distribution and its second moment. To measure $N_{0}$ we count the atoms within the central peak rising above the smooth thermal distribution.

From the measured $N(t)$ alone we calculate the equilibrium $T_{\mathrm{c}}(t)$ (ref. 2). From $N(t)$ and $E(t)$ we calculate the equilibrium intensive thermodynamic variables $\mu^{\mathrm{eq}}(t)$ and $T^{\mathrm{eq}}(t)$, and the equilibrium number of condensed atoms, $N_{0}^{\text {eq }}(t)$ (refs 2,20,21); in these calculations $N_{0}^{\mathrm{eq}}>0$ if and only if $T^{\mathrm{eq}}<T_{\mathrm{c}}$. For comparison, we also directly fit a temperature $T^{f}$ to the wings of the momentum distribution. In addition, supposing only that the two components are separately in equilibrium, from the measured $N_{0}$ and $N^{\prime}$ we calculate $\mu_{0}$ and $\mu^{\prime}$. (For theoretical details see Supplementary Information.)
At $83 a_{0}$ (Fig. 2a) we find excellent agreement between the measured $N_{0}$ and the $N_{0}^{\mathrm{eq}}$ predicted without any free parameters. The BEC vanishes exactly at the equilibrium critical time $t_{\mathrm{c}}$ (dotted green line), at which $T^{\mathrm{eq}}=T_{\mathrm{c}}$. Note that the dashed red lines show the experimental bounds on the time $\bar{t}$ when the BEC vanishes. The separately calculated $\mu_{0}, \mu^{\prime}$ and $\mu^{\mathrm{eq}}$ are all consistent and we have also checked that the fitted $T^{f}$ coincides with the calculated $T^{\mathrm{eq}}$. All this gives us full confidence in our equilibrium calculations.

At $5 a_{0}$ (Fig. 2b) we observe strikingly different behaviour. The BEC now survives much longer than it would in true equilibrium; $\bar{t}-t_{\mathrm{c}} \approx 40 \mathrm{~s}$. We also see that $\mu_{0}$ and $\mu^{\prime}$ diverge from each other for $t>t_{\mathrm{c}}$, so the system is moving away from the global phase equilibrium rather than towards it. The observed superheating can 


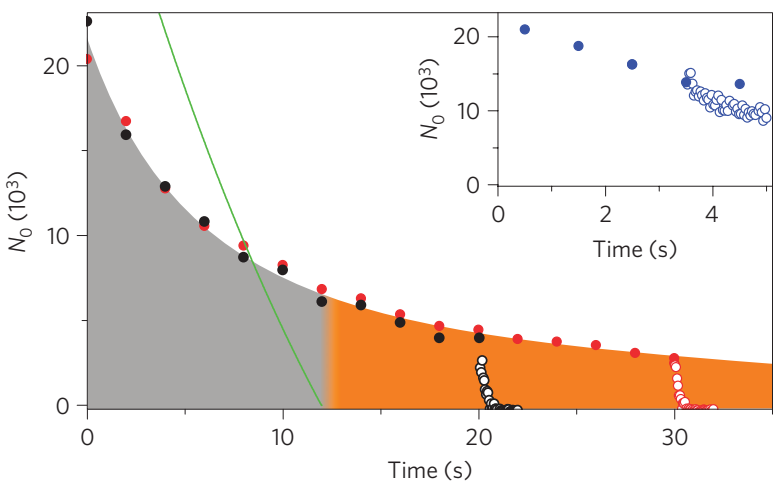

Figure 3 | Quenching the superheated Bose-condensed gas. Filled symbols show the evolution of $N_{0}$ at $a=3 a_{0}$, the green solid line shows $N_{0}^{\text {eq }}$ and orange shading indicates the superheated regime. Open symbols show the rapid decay of the $\mathrm{BEC}$ after it is strongly coupled to the thermal bath by an interaction quench to $a=62 a_{0}$ at time $t_{q}$. We show two experimental series in which $t_{q}=20 \mathrm{~s}$ (black) and $30 \mathrm{~s}$ (red). Inset: an interaction quench at a time when $T<T_{\mathrm{c}}$ and $N_{0}^{\mathrm{eq}}>0$ does not kill the BEC. (Note that here we make an even stronger quench, to $100 a_{0}$.)

thus not be understood as just a transient effect. (Note that $\mu_{0}-\mu_{\mathrm{c}}$ is always very small owing to weak interactions.)

At $5 a_{0}$ the gas is not in global phase equilibrium, but it is still a good approximation to view its two components as two equilibrium subsystems at the same (kinetic) temperature, as in Fig. 1b. We have checked that the momentum distribution in the non-condensed component is still fitted well by a thermal distribution, with $T^{f}$ always within $10 \%$ of the calculated $T^{\text {eq }}(N, E)$ (see Supplementary Information). For the BEC, in a weakly interacting gas the equilibrium relation $\mu_{0}\left(N_{0}\right)$ relies on the macroscopic occupation of a single quantum state ${ }^{1}$, rather than on global equilibrium. Moreover, even for the lowest-energy collective modes we estimate the Landau damping time to be $<1 \mathrm{~s}$ (refs 16,17; see Supplementary Information), that is, much shorter than the characteristic timescale of our experiments. Thus, although this distribution is not directly measurable, we expect the distribution of collective excitations in the $\mathrm{BEC}$ to be characterized by a temperature $T_{0}$ that is also close to $T^{\mathrm{eq}} \approx T^{f}$.

These conclusions hold for any $a \gtrsim 1 a_{0}$ (see Supplementary Information). Exactly at $a=0$ our theoretical picture does break down, because the Landau damping rate vanishes and the BEC has no equilibrium features; the two components are simply completely decoupled. Bearing this small caveat in mind, from here on we refer to $T_{0} \approx T^{f} \approx T^{\mathrm{eq}}$ simply as the temperature of the system $T$.

A way to directly see that the gas is superheated is to suddenly increase the coupling of the BEC to the thermal bath. In Fig. 3 we show the results of two experimental series in which $a$ is quenched (within $10 \mathrm{~ms}$ ) from $3 a_{0}$ to $62 a_{0}$ at different times in the superheated regime. The filled (open) symbols show $N_{0}$ measured before (after) the quench. The small $\Gamma_{0}$ is almost unaffected by the change in $a$, and the sudden $N_{0}$ decay is due to the increase in $\kappa$ (Fig. 1b). For reference, the green line shows the calculated $N_{0}^{\text {eq }}$ at $3 a_{0}$ and orange shading indicates the superheated regime. As shown in the inset of Fig. 3, we have checked that an interaction quench at $t<t_{\mathrm{c}}$ (that is, when $T<T_{\mathrm{c}}$ and $N_{0}^{\text {eq }}>0$ ) does not kill the BEC.

As shown in Fig. 4, we have explored the limits of superheating for a range of interaction strengths, including small negative values of $a$. For $a<0$, a BEC is stable against collapse only for $N_{0}<-C / a$, with $C \approx 2 \times 10^{4} a_{0}$ for our trap parameter ${ }^{22-24}$. However, after $N_{0}$ drops below this critical value, at small $|a|$ it decays slowly.

In Fig. 4a we plot the highest temperature at which we still observe a BEC, $\bar{T} \equiv T(t=\bar{t})$, scaled to the equilibrium $T_{\mathrm{c}}$ at

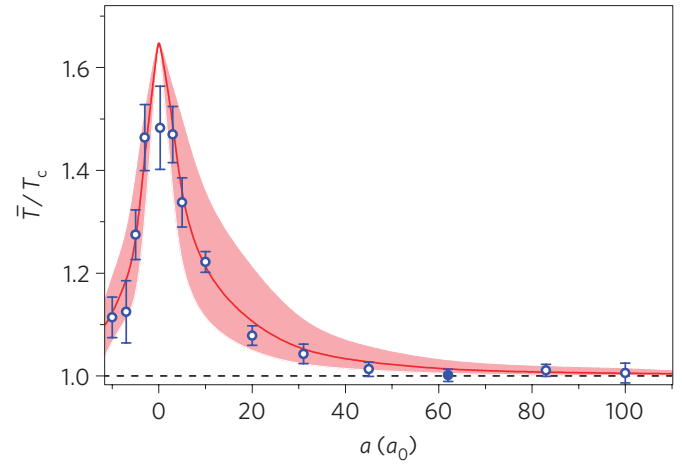

b

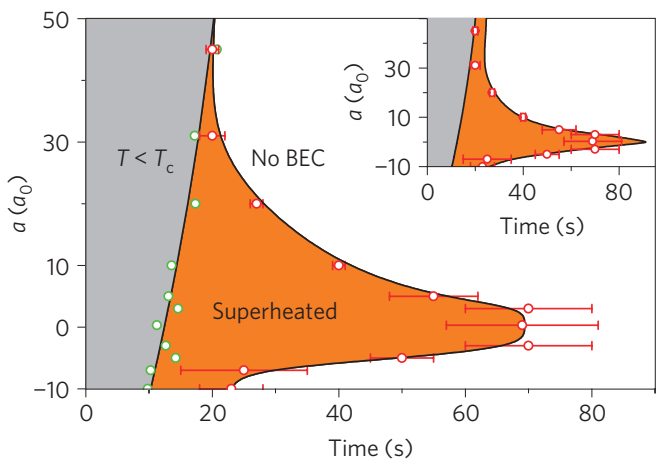

Figure 4 | Limits of superheating. a, The highest temperature at which we observe a BEC, $\bar{T}$, scaled to the equilibrium $T_{\mathrm{C}}$. Close to $a=0$ the BEC survives up to $\approx 1.5 T_{\mathrm{c}}$. The red line shows the results of our numerical calculations, with the shaded area indicating the theoretical uncertainty. Experimental error bars are statistical. The point at $62 a_{0}$ is fixed to unity by the absolute atom number calibration ${ }^{2,27} . \mathbf{b}$, Temporal phase diagram. For each value of $a$ we plot the equilibrium $t_{c}$ (green points) and the time $\bar{t}$ at which the BEC actually vanishes (red points). The $\bar{t}$ errors correspond to dashed lines in Fig. 2 and the uncertainty in $t_{\mathrm{c}}$ is indicated by the scatter of points. Solid curves are spline fits to the data. For $a \approx 0$ the BEC survives in the superheated regime for a whole minute. Inset: numerically calculated phase diagram, with $\bar{t}$ data overlaid.

the same $N$. For $a \rightarrow 0$, the BEC survives up to $T \approx 1.5 T_{\mathrm{c}}$. (For comparison, this is analogous to superheated water at $280^{\circ} \mathrm{C}$.)

In Fig. $4 \mathrm{~b}$ we reconstruct the temporal phase diagram of our non-equilibrium gas. Here, a horizontal cut through the graph corresponds to a time series such as shown in Fig. 2. For each $a$, we plot the measured $\bar{t}$ (red points) and the equilibrium $t_{\mathrm{c}}$ (green points). The solid curves are spline fits to the data. The width of the orange-shaded region corresponds to the time that the BEC survives in the superheated regime. For $a \approx 0$ this region spans a whole minute.

The phase diagram in Fig. $4 \mathrm{~b}$ is measured by always starting with $N_{0} \approx 2 \times 10^{4}$. In general, non-equilibrium behaviour can strongly depend on the initial conditions. However, we find that $\bar{t}$ is essentially constant (within experimental errors) for initial $N_{0}$ in the range $(1-5) \times 10^{4}$. The primary reason for this is that the three-body contribution to $\Gamma_{0}$ grows with $N_{0}$; this leads to self-stabilization of the condensed atom number on timescales much shorter than $\bar{t}$.

We theoretically reproduce our non-equilibrium observations using a two-component model directly corresponding to Fig. $1 \mathrm{~b}$. Starting with the measured initial $N_{0}$, we numerically simulate the evolution of a BEC coupled to a thermal bath characterized by $\mu^{\prime}(t)$. To do this we calculate $\Gamma_{0}$ from our experimental parameters, and for $\kappa$ we use the form ${ }^{25}$

$$
\kappa=A \gamma_{\mathrm{el}} N_{0}\left[\mathrm{e}^{\beta\left(\mu_{0}-\mu_{\mathrm{c}}\right)}-\mathrm{e}^{\beta\left(\mu^{\prime}-\mu_{\mathrm{c}}\right)}\right]
$$


Here $\gamma_{\mathrm{el}} \propto a^{2}$ is the elastic collision rate and $A$ is a dimensionless coefficient. The largest uncertainty in our calculations comes from the theoretical uncertainty in $A \approx 1-10$ (ref. 26). (For details see Supplementary Information.)

In Fig. 4a we show the calculated $\bar{T} / T_{\mathrm{c}}$. The red line corresponds to $A=3$ and the shaded area to the range $A=1-10$. The calculation generally captures our experimental observations well. With $A=3$ we obtain quantitative agreement with the data, except exactly at $a=0$, where the model is not valid. In the inset of Fig. $4 \mathrm{~b}$ we show the calculated temporal phase diagram, with $A=3$, together with the experimental $\bar{t}$ data. Again the general features of the diagram are captured well for $a \neq 0$.

The success of our calculations supports a conceptually simple way to think about dynamical non-equilibrium effects near a continuous phase transition. Extending the BEC lifetime by tuning interactions could also have practical benefits for precision measurements and quantum information processing.

Received 3 September 2012; accepted 14 February 2013; published online 24 March 2013; corrected online 28 March 2013

\section{References}

1. Dalfovo, F., Giorgini, S., Pitaevskii, L. P. \& Stringari, S. Theory of Bose-Einstein condensation in trapped gases. Rev. Mod. Phys. 71, 463-512 (1999).

2. Smith, R. P., Campbell, R. L. D., Tammuz, N. \& Hadzibabic, Z. Effects of interactions on the critical temperature of a trapped Bose gas. Phys. Rev. Lett. 106, 250403 (2011).

3. Polkovnikov, A., Sengupta, K., Silva, A. \& Vengalattore, M. Colloquium: Nonequilibrium dynamics of closed interacting quantum systems. Rev. Mod. Phys. 83, 863-883 (2011).

4. Kinoshita, T., Wenger, T. \& Weiss, D. S. A quantum Newton's cradle. Nature 440, 900-903 (2006).

5. Winkler, K. et al. Repulsively bound atom pairs in an optical lattice. Nature 441, 853-856 (2006).

6. Sadler, L. E., Higbie, J. M., Leslie, S. R., Vengalattore, M. \& Stamper-Kurn, D. M. Spontaneous symmetry breaking in a quenched ferromagnetic spinor Bose-Einstein condensate. Nature 443, 312-315 (2006).

7. Hofferberth, S., Lesanovsky, I., Fischer, B., Schumm, T. \& Schmiedmayer, J. Non-equilibrium coherence dynamics in one-dimensional Bose gases. Nature 449, 324-327 (2007).

8. Haller, E. et al. Realization of an excited, strongly correlated quantum gas phase. Science 325, 1224-1227 (2009).

9. Guzman, J. et al. Long-time-scale dynamics of spin textures in a degenerate $F=1{ }^{87} \mathrm{Rb}$ spinor Bose gas. Phys. Rev. A 84, 063625 (2011).

10. Cheneau, M. et al. Light-cone-like spreading of correlations in a quantum many-body system. Nature 481, 484-487 (2012).

11. Trotzky, S. et al. Probing the relaxation towards equilibrium in an isolated strongly correlated one-dimensional Bose gas. Nature Phys. 8, 325-330 (2012).

12. Mark, M. J. et al. Preparation and spectroscopy of a metastable Mott-insulator state with attractive interactions. Phys. Rev. Lett. 108, 215302 (2012).
13. Smith, R. P., Beattie, S., Moulder, S., Campbell, R. L. D. \& Hadzibabic, Z. Condensation dynamics in a quantum-quenched Bose gas. Phys. Rev. Lett. 109, 105301 (2012).

14. Gring, M. et al. Relaxation and pre-thermalization in an isolated quantum system. Science 337, 1318-1322 (2012).

15. Mathey, L. \& Polkovnikov, A. Light cone dynamics and reverse Kibble-Zurek mechanism in two-dimensional superfluids following a quantum quench. Phys. Rev. A 81, 033605 (2010).

16. Fedichev, P. O., Shlyapnikov, G. V. \& Walraven, J. T. M. Damping of low-energy excitations of a trapped Bose-Einstein condensate at finite temperatures. Phys. Rev. Lett. 80, 2269-2272 (1998).

17. Pethick, C. \& Smith, H. Bose-Einstein Condensation in Dilute Gases (Cambridge Univ. Press, 2002).

18. Campbell, R. L. D. et al. Efficient production of large ${ }^{39} \mathrm{~K}$ Bose-Einstein condensates. Phys. Rev. A 82, 063611 (2010)

19. Roati, G. et al. ${ }^{39} \mathrm{~K}$ Bose-Einstein condensate with tunable interactions. Phys. Rev. Lett. 99, 010403 (2007).

20. Tammuz, N. et al. Can a Bose gas be saturated? Phys. Rev. Lett. 106, 230401 (2011).

21. Smith, R. P. \& Hadzibabic, Z. Effects of interactions on Bose-Einstein condensation of an atomic gas. Preprint at http://arxiv.org/abs/1203. 2063 (2012).

22. Ruprecht, P. A., Holland, M. J., Burnett, K. \& Edwards, M. Time-dependent solution of the nonlinear Schrödinger equation for Bose-condensed trapped neutral atoms. Phys. Rev. A 51, 4704-4711 (1995).

23. Gerton, J. M., Strekalov, D., Prodan, I. \& Hulet, R. G. Direct observation of growth and collapse of a Bose-Einstein condensate with attractive interactions. Nature 408, 692-695 (2000).

24. Donley, E. A. et al. Dynamics of collapsing and exploding Bose-Einstein condensates. Nature 412, 295-299 (2001).

25. Gardiner, C. W., Zoller, P., Ballagh, R. J. \& Davis, M. J. Kinetics of Bose-Einstein condensation in a trap. Phys. Rev. Lett. 79, 1793-1796 (1997).

26. Gardiner, C. W., Lee, M. D., Ballagh, R. J., Davis, M. J. \& Zoller, P. Quantum kinetic theory of condensate growth: Comparison of experiment and theory. Phys. Rev. Lett. 81, 5266-5269 (1998).

27. Smith, R. P., Tammuz, N., Campbell, R. L. D., Holzmann, M. \& Hadzibabic, Z. Condensed fraction of an atomic Bose gas induced by critical correlations. Phys. Rev. Lett. 107, 190403 (2011).

\section{Acknowledgements}

We thank S. Beattie and S. Moulder for experimental assistance. This work was supported by EPSRC (Grant No. EP/K003615/1), the Royal Society, AFOSR, ARO and DARPA OLE.

\section{Author contributions}

All authors contributed extensively to this work.

\section{Additional information}

Supplementary information is available in the online version of the paper. Reprints and permissions information is available online at www.nature.com/reprints. Correspondence and requests for materials should be addressed to R.P.S.

\section{Competing financial interests}

The authors declare no competing financial interests. 